\title{
Carrier Density Control by Illumination in Surface Doped, $p$-Type (Cd,Mn)Te Quantum Wells
}

\author{
W. Maślana ${ }^{a, b}$, P. Kossacki ${ }^{a, b}$, J.A. GaJ $^{a}$, D. Ferrand $^{b}$, \\ M. Bertolini ${ }^{b}$, S. Tatarenko $^{b}$, J. Cibert $^{c}$, M. Kutrowski $^{d}$ \\ AND T. WOJTOWICZ ${ }^{d}$ \\ ${ }^{a}$ Institute of Experimental Physics, Warsaw University \\ Hoża 69, 00-681 Warsaw, Poland \\ ${ }^{b}$ Laboratoire de Spectrométrie Physique \\ CNRS et Université Joseph Fourier - Grenoble \\ B.P. 87, 38402 Saint Martin d'Héres Cedex, France \\ ${ }^{c}$ Laboratoire Louis Néel, CNRS, B.P. 166, 38042 Grenoble Cedex, France \\ ${ }^{d}$ Institute of Physics, Polish Academy of Sciences \\ al. Lotników 32/46, 02-668 Warsaw, Poland
}

\begin{abstract}
We report both decrease and increase in the $2 \mathrm{D}$ carrier gas density in a simple $(\mathrm{Cd}, \mathrm{Mn}) \mathrm{Te} /(\mathrm{Cd}, \mathrm{Mg}) \mathrm{Te}$ heterostructure with $(\mathrm{Cd}, \mathrm{Mn}) \mathrm{Te}$ quantum well. The two effects were achieved by light with different photon energies. The quantum wells were $10 \mathrm{~nm}$ wide with 2D hole gas supplied by surface states. For the sample with $25 \mathrm{~nm}$ cap layer thickness, it was possible to tune the hole gas concentration from almost empty well (hole density below $1 \times 10^{10} \mathrm{~cm}^{-2}$ ) to $45 \times 10^{10} \mathrm{~cm}^{-2}$. The illumination with $425 \mathrm{~nm}$ wavelength almost doubled the hole gas density from the initial $24 \times 10^{10} \mathrm{~cm}^{-2}$. The depletion mechanism was most effective for illumination with the orange (575 nm) light.
\end{abstract}

PACS numbers: 75.50.Pp, 78.67.De, 61.72.Vv

\section{Introduction}

The optical properties of $2 \mathrm{D}$ carrier gas are widely studied. They were used as a probe of carrier system by $[1,2]$. The optical experiments are essential tool in studies of charged excitons [3-6], as well as carrier induced ferromagnetism in quantum wells (QWs) [7, 8]. When optical experiments are used, it is important 
to understand whether the light used as a probe of the properties of the sample may influence significantly its state. In semimagnetic heterojunctions made of III-V semiconductors studied by [9] the light was used to increase the hole gas concentration. In this case photocreated hole density was the necessary condition for the observation of ferromagnetic ordering.

In the structures containing a single $\mathrm{CdTe} /(\mathrm{Cd}, \mathrm{Zn}, \mathrm{Mg}) \mathrm{Te}$ modulation $n$-doped (iodine or aluminium donors) quantum well, blue laser light was found to increase $[10,11]$ the electron density inside quantum well. On the other hand, in similar structures, illumination with blue light was found to either decrease or increase the electron concentration in the quantum well [12], depending on the thickness of the doping layer embedded in the structure.

In $p$-doped CdTe-based single QW structures, blue light illumination was found to decrease the hole density in case of nitrogen doping [4]. In this work we study the possibilities of the carrier density control in quantum well through illumination by light of different wavelengths.

\section{Samples and carrier density determination}

Samples were grown by molecular beam epitaxy (MBE) technique at CEA-CNRS laboratory in Grenoble. In this work we used two samples grown on different substrates: $\mathrm{Cd}_{0.88} \mathrm{Zn}_{0.12}$ Te and $\mathrm{Cd}_{0.96} \mathrm{Zn}_{0.04}$ Te. Both contained a $10 \mathrm{~nm}$ wide QW made of $\mathrm{Cd}_{1-x} \mathrm{Mn}_{x}$ Te. The quantum wells contained 2D hole gas supplied by surface states [13]. The QW was embedded between $\mathrm{Cd}_{0.7} \mathrm{Zn}_{0.08} \mathrm{Mg}_{0.22} \mathrm{Te}$ barriers in case of $12 \% \mathrm{Zn}$ substrate (sample $A$ ) and $\mathrm{Cd}_{0.78} \mathrm{Mg}_{0.22}$ Te for the sample $B$ grown on the $4 \% \mathrm{Zn}$ substrate, so that the whole structure could be grown coherently strained to the substrate. The $12 \% \mathrm{Zn}$ substrate is transparent at QW transition energies, enabling us to perform transmission measurements on sample $A$. The thickness of the top barrier (cap layer) was $25 \mathrm{~nm}$ which gives the maximum hole density in quantum wells close to the surface [13]. The Moss-Burstein shift measurements [4] yielded hole gas density of $30 \times 10^{10} \mathrm{~cm}^{-2}$ in sample $A$ and $24 \times 10^{10} \mathrm{~cm}^{-2}$ in sample $B$.

In sample $A$ a nitrogen doped layer was placed between the quantum well and the substrate, at distance of $100 \mathrm{~nm}$ from the quantum well. Its purpose is to screen the quantum well from any possible charge transfer from sources different than the surface states.

For carrier density determination we used the results of a previous work performed on nitrogen modulation doped (Cd,Mn)Te/(Cd,Mg)Te QW [4]. The acceptor layer was placed $20 \mathrm{~nm}$ from the quantum well and the obtained hole concentration in the quantum well was $24 \times 10^{10} \mathrm{~cm}^{-2}$. The hole gas density was determined from the Moss-Burstein shift measurements. It was found that the blue light of the photon energy larger than the band gap of the barrier material can decrease the hole gas density in the quantum well. As a result changes of absorption intensity were observed for both charged and neutral excitons. The 

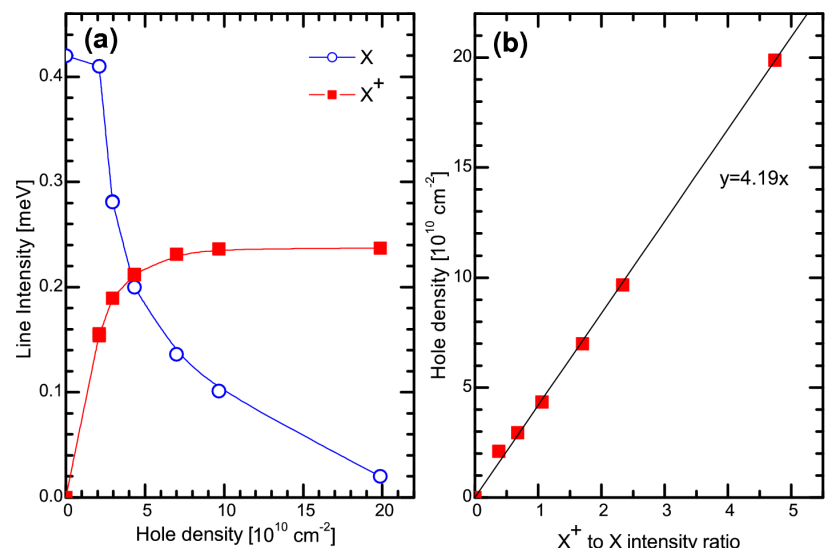

Fig. 1. (a) Neutral and charged exciton integrated absorption line intensities. (b) Hole gas density as a function of charged to neutral exciton intensity ratio computed for the data presented in part (a).

intensity of neutral exciton transition decreases while that of the charged one increases with the increase in hole gas density. This can be seen in Fig. 1a where values of integrated intensities at different hole densities are presented. We were able to establish that the ratio of integrated intensities can be used as a sample independent measure of the hole gas density. This approach is shown in Fig. 1b where we plot the ratio intensities of neutral and charged exciton lines determined by [4] versus independently determined hole gas density (from the Moss-Burstein shift). The dependence can be effectively described by a straight line. This allows us to deduce the carrier density from a single transmission spectrum.

\section{Experimental results}

We shall focus on the sample $A$ where transmission measurements were possible due to $\mathrm{Cd}_{0.88} \mathrm{Zn}_{0.12}$ Te substrate. The arrangement of experimental setup was similar to the one used in [4]. The light used for transmission measurement was directed from the back side of the sample. The thick substrate layer acts as a high pass spectral filter, absorbing all of the light below $745 \mathrm{~nm}$ wavelength preventing heating of the quantum well by the transmitted light. Additionally, it prevented absorption by the barrier material (which has a significantly larger band gap), thus we expected that the transmitted light would not affect the carrier density.

The additional illumination provided by the tungsten lamp was applied on the side of the sample with the heterostructure. In order to check whether the impact of the light on the carrier density has a different spectral response than in the previously studied case [4], the experimental setup was modified with respect to paper [4]. The illumination line was modified in order to replace spectral filter by a monochromator. The slits were set to optimize the amount of transmitted light and provided a beam of $3 \mathrm{~nm}$ spectral width. For different illumination wavelengths, 
ranging from the $750 \mathrm{~nm}$ to $460 \mathrm{~nm}$, transmission spectra were recorded. The sample temperature was set to $1.7 \mathrm{~K}$.

The results are presented in Fig. 2. Part (a) presents optical density spectra, integrated line intensities are presented in part (b). For illumination light at wavelength between $750 \mathrm{~nm}$ and $615 \mathrm{~nm}$ there seems to be no effect on the transmission spectra. When the illumination wavelength is further decreased below $610 \mathrm{~nm}$ a second line appears in the optical density spectra. It has been identified as a neutral exciton transition. After an initial rise, the neutral exciton intensity reaches a maximum at about $590 \mathrm{~nm}$, and then exhibits a slow decrease. The change of neutral exciton line intensity is accompanied by small changes of charged exciton intensity and a change of charged exciton dissociation energy (typical effects associated with the variation of hole gas density) [4].
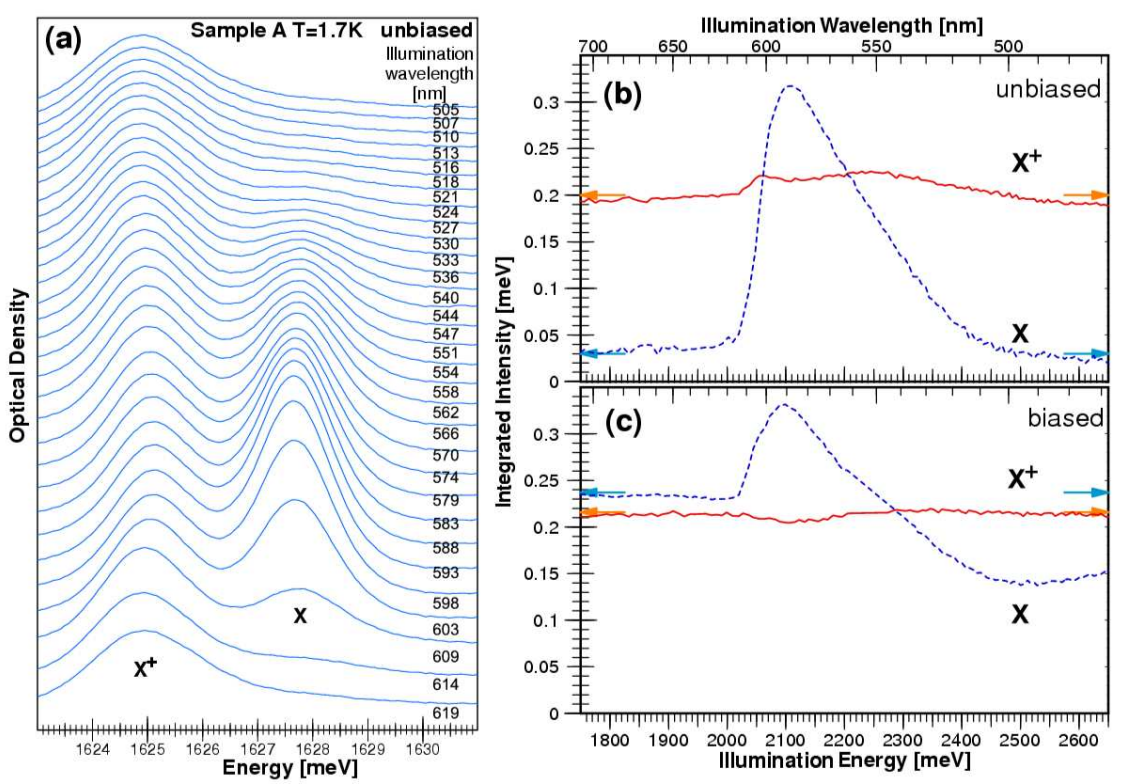

Fig. 2. The effect of the illumination on the absorption. (a) Optical density spectra. (b),(c) Integrated intensities of optical transitions present in the transmission as a function of illumination photon energy. Solid line denotes intensity of charged exciton, dashed one denotes intensity of exciton. The results in part (c) were obtained with additional optical bias (illumination $590 \mathrm{~nm}$ of moderate power).

Only a part of the studied range of the hole gas density lies in the area where the excitonic transition is pronounced. This is most unfortunate as we would like to analyze changes of hole gas density through the intensity ratio of both transitions. Therefore for the purpose of increasing the sensitivity of experiment an additional optical bias was applied in order to reduce the initial density of hole 
gas present in the quantum well. It was achieved by adding to the setup another illumination line. Now there were two of them: one of tunable wavelength with monochromator and the second line with fixed wavelength determined by a narrow band-pass (interference) spectral filter of $590 \mathrm{~nm}$ center wavelength. The $590 \mathrm{~nm}$ wavelength was chosen due to the maximal efficiency of the quantum well depletion as seen in Fig. 2b. The intensity of orange $(590 \mathrm{~nm})$ light illumination was tuned ensuring that both excitonic transitions were observed for the whole range of illumination wavelengths provided by the monochromator. The initial hole gas density in case of this measurement was reduced to $3.8 \times 10^{10} \mathrm{~cm}^{-2}$. The results of the measurements with two illuminations are presented in Fig. 2c. Apparently the additional illumination not only enhanced the sensitivity of experiment, but it also allowed us to observe additional processes occurring for illumination with higher photon energies.

The intensities of neutral and charged excitons were converted into the hole gas density calibrated according to Fig. 1b. The results are presented in Fig. 3a. Apparently, the strong changes of neutral exciton intensity seen in the experiment with optical bias do not result in high variation of absolute hole gas density when compared to the unbiased case. However, the experimental results obtained without bias for high hole densities exhibit significant noise. This is the result of worse determination of line intensities when neutral exciton transition is weak. The lowering of initial carrier density by additional bias improves the accuracy.

In order to compare the biased and unbiased experiments it is more convenient to discuss the changes of hole gas density with respect to their initial values. In the experiment without bias we can consider as an initial value the hole density before any illumination, in biased case we take for the initial value the hole gas density with only bias illumination applied (values marked by arrows in Fig. 3a). The values of the hole density variations are presented in Fig. 3b.

For the unbiased experiment the most pronounced feature is the drop of hole gas density. It is still visible in experiment with bias although it is less spectacular. The effective reduction of hole gas density starts for the illumination photon energy higher than $2010 \mathrm{meV}$. This critical energy for depletion is related to the band gap of barrier material. It can be seen in the inset in Fig. 3b where the photoluminescence (PL) spectrum of the barriers is presented with the same energy scale as the main graph.

In both cases the depletion reaches a maximum at $2100 \mathrm{meV}$. In both experiments we have achieved almost the same minimal hole gas density. It was $2.5 \times 10^{10} \mathrm{~cm}^{-2}$ with bias and $3 \times 10^{10} \mathrm{~cm}^{-2}$ without. This indicates that additional bias power was very close to the power of light passing through the monochromator. Only a small reduction of hole density for almost doubled power of light indicates that we approached the maximal efficiency of depletion with illumination. The effect of illumination power (at constant wavelength) was analyzed in [4] and similar effects are reported under strong illumination. 


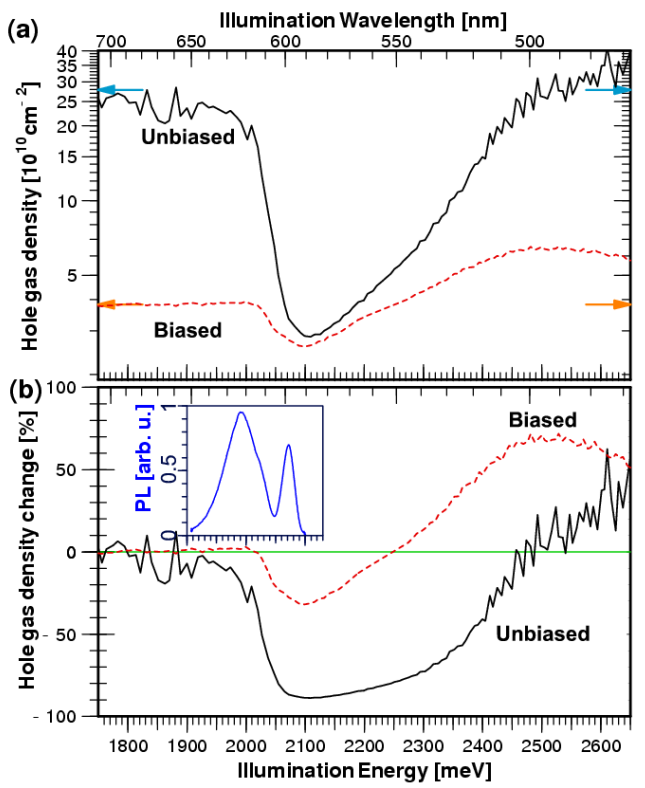

Fig. 3. (a) Hole gas density determined from ratio of charged to neutral exciton oscillator strength as a function of illumination photon energy. Solid line denotes results for the single illumination experiment, dashed line presents results for the experiment with $590 \mathrm{~nm}$ illumination bias. The vertical scale is logarithmic. (b) Dependence of hole gas density change on the illumination photon energy. Continuous line denotes results for the single illumination experiment, dashed line presents results for the experiment with $590 \mathrm{~nm}$ illumination bias. The inset presents photoluminescence spectra from the barriers under ion Ar laser excitation - the photon energy scale is the same as for the main plot.

After reaching the minimal value the concentration increases for the increasing photon energies. One could expect it as a natural consequence of the decrease in illumination power due to the transmission characteristic of the applied monochromator and the spectral characteristic of the light emitted by the tungsten bulb used in the experiment. There are several features in Fig. 3b indicating that such a simple explanation is insufficient. Firstly, for both experimental curves the rise of hole density for high illumination photon energies surpasses the initial hole density. Secondly, the rise of hole gas density is much steeper for the sample with additional bias despite the fact that the changes of illuminating light power are the same for both curves.

The effect of power is probably dominating only in the range between $2100 \mathrm{meV}$ and $2250 \mathrm{meV}$ in unbiased experiment because for the $50 \%$ decrease in illumination power we have only $10 \%$ rise of hole gas density which is comparable to the results of [4] for variation of illumination power at constant wavelength. 
Steep slope of hole gas density change for both curves at photon energies higher than $2300 \mathrm{meV}$ is a result of competition between the depletion mechanism and another mechanism leading to the increase in hole gas density. For high photon energies the increase in hole gas density is favored.

The additional optical bias besides reducing the hole gas density in the quantum well also significantly affects the spectral response to the illumination. The maximal depletion is achieved at the same illumination wavelength as without bias. However, the depletion mechanism was neutralized for much smaller photon energies $(2250 \mathrm{meV})$. Further increase in photon energy results in actual increase in hole gas density beyond initial value. The increase reaches maximum of $70 \%$ at $2480 \mathrm{meV}$. The saturation of increase is probably caused by the significant decrease in the illumination power as compared to the initial bias. For the unbiased experiment even such small intensity turns out sufficient to increase the hole density above the initial value, as in this case the depletion mechanism is not aided by additional light.

\section{Carrier control mechanism in surface doped samples}

The decrease in the hole gas density is possible only for the illumination with photons of higher energy than the energy band gap of the barrier material. Such behavior is in agreement with the discussion and the model used for the nitrogen doped samples [4]. The main mechanism remains as even the distances of acceptor sites from the quantum well are similar in both cases. The input of the screening layer to these processes is negligible as it is too distant from the quantum well. This has been confirmed by the observations of the time necessary to return to the nominal hole gas density after shutting down the additional illumination depleting the quantum well. For the studied sample $A$ it was relatively quick, as the change from depleted to nominal concentration occurred in time shorter than 1 second. For the samples with thicker cap layers and structure identical to sample $A$ this time is significantly elongated to about $2 \mathrm{~s}$ at $40 \mathrm{~nm}$ cap and about $5 \mathrm{~s}$ in the case of $60 \mathrm{~nm}$ cap layer.

The understanding of the mechanism responsible for increase in the hole gas density is more difficult. One possibility is a decrease in the electron trapping efficiency by the QW, aided by an enhancement of the hole tunneling probability at high kinetic energies. The electrons penetrate deep in the sample while the holes tunnel into the QW. When the photocreated electron penetrates deep into the sample structure, it has to pass over the barrier of nitrogen acceptors.

The lowering of the threshold energy for this process in the experiment with optical bias may suggest that the bias saturates the acceptor sites and thus lowers the barrier for the electrons in the conduction band. Therefore one may expect that the absence of the nitrogen doping layer may strengthen the effects increasing the carrier density change under blue illumination. Indeed, for the sample $B$, with identical $25 \mathrm{~nm}$ layer thickness but without the screening layer embedded in the 
structure, the additional illumination with $425 \mathrm{~nm}$ wavelength allowed to almost double the hole gas density reaching $45 \times 10^{10} \mathrm{~cm}^{-2}$ from the initial $24 \times 10^{10} \mathrm{~cm}^{-2}$. In this case the depletion mechanism was also effective. It allowed to almost empty the quantum well from the carriers with the yellow $(575 \mathrm{~nm})$ light illumination.

\section{Summary}

The structures using the surface acceptor states exhibit a unique sensitivity to the light. Depending on the applied illumination wavelength it is possible either to increase or to decrease the carrier density in the quantum well in the same structure.

\section{References}

[1] M.S. Skolnick, K.J. Nash, M.K. Saker, S.J. Bass, P.A. Claxton, J.S. Roberts, Appl. Phys. Lett. 50, 1885 (1987).

[2] M.S. Skolnick, J. M. Rorison, K.J. Nash, D.J. Mowbray, P.R. Tapster, S.J. Bass, A.D. Pitt, Phys. Rev. Lett. 58, 2130 (1987).

[3] K. Kheng, R.T. Cox, Y.M. d'Aubigné, F. Bassani, K. Saminadayar, S. Tatarenko, Phys. Rev. Lett. 71, 1752 (1993).

[4] P. Kossacki, J. Cibert, D. Ferrand, Y.M. d'Aubigné, A. Arnoult, A. Wasiela, S. Tatarenko, J.A. Gaj, Phys. Rev. B 60, 16018 (1999).

[5] P. Płochocka, P. Kossacki, W. Maślana, J. Cibert, S. Tatarenko, C. Radzewicz, J.A. Gaj, Phys. Rev. Lett. 92, 177402 (2004).

[6] R.T. Cox, R.B. Miller, K. Saminadayar, T. Baron, Phys. Rev. B 69, 235303 (2004).

[7] A. Haury, A. Wasiela, A. Arnoult, J. Cibert, S. Tatarenko, T. Dietl, Y.M. d'Aubigné, Phys. Rev. Lett. 79, 511 (1997).

[8] P. Kossacki, D. Ferrand, M. Goryca, M. Nawrocki, W. Pacuski, W. Maślana, S. Tatarenko, J. Cibert, Physica E 32, 454 (2006).

[9] S. Koshihara, A. Oiwa, M. Hirasawa, S. Katsumoto, Y. Iye, C. Urano, H. Takag, H. Munekata, Phys. Rev. Lett. 78, 4617 (1997).

[10] V. Huard, R.T. Cox, K. Saminadayar, A. Arnoult, S. Tatarenko, Phys. Rev. Lett. 84, 187 (2000).

[11] V. Huard, R.T. Cox, K. Saminadayar, C. Bourgognon, A. Arnoult, J. Cibert, S. Tatarenko, Physica E 6, 161 (2000).

[12] M. Kutrowski, Ph.D. thesis, Institute of Physics, Polish Academy of Sciences, Warszawa 2001.

[13] W. Maślana, P. Kossacki, M. Bertolini, H. Boukari, D. Ferrand, S. Tatarenko, J. Cibert, J.A. Gaj, Appl. Phys. Lett. 82, 1875 (2003). 